\title{
Commensal Lactobacillus Suppress Colon Tumorigenesis and Progression by Modulation of Sphingosine 1-Phosphate Signaling in Mice
}

\section{Fuqiang Xu}

Institute of Modern Physics $\mathbb{}$ Chinese Academy of Sciences

Qiaoqiao Li

Institute of Modern Physics $₫$ Chinese Academy of Sciences

Shuyang Wang ( $\nabla$ wangsy@impcas.ac.cn )

Institute of Modern Physics $\llbracket$ Chinese Academy of Sciences

Miaoyin Dong

Institute of Modern Physics $\llbracket$ Chinese Academy of Sciences

Guoqing Xiao

Institute of Modern Physics $\llbracket$ Chinese Academy of Sciences

Jin Bai

Institute of Modern Physics $\llbracket$ Chinese Academy of Sciences

Junkai Wang

Northwest Normal University

Xisi Sun

Institute of Modern Physics $\llbracket$ Chinese Academy of Sciences

\section{Research Article}

Keywords: Commensal Lactobacillus, Colon cancer, Gut microbiota, Metabolites, Sphingosine 1phosphate signaling

Posted Date: February 11th, 2022

DOI: https://doi.org/10.21203/rs.3.rs-1322563/v1

License: (c) (i) This work is licensed under a Creative Commons Attribution 4.0 International License. Read Full License 


\section{Abstract}

Background: Gut microbiota and their secreted metabolites influence the initiation, progression, and treatment responsiveness of colon cancer. Microbiota-based therapy regulated by probiotics has been considered as an effective strategy for prevention and therapeutic of colon cancer, whereas the antitumor mechanisms influenced by microbiota and their metabolites with intervention of probiotic remain further investigated.

Results: Here, we investigated the preventive and therapeutic efficiency of commensal Lactobacillus in subcutaneous tumor models, and the underlying antitumor mechanism through the alteration of gut microbiota and their metabolites regulated by commensal Lactobacillus. Interestingly, we found that tumor formation rate in subcutaneous tumor models reduced $86.21 \%$ and $82.76 \%$, in comparison to tumor controls when administrated with living or inactivated Lactobacillus (JY300-8 and JMR-01) for 20 days in advance. Subsequently, continuously oral administration for living or inactivated commensal Lactobacillus suppressed significantly the growth of tumor, and the tumor volumes declined $65.2 \%$ and $61.18 \%$, respectively. Furthermore, microbiome and metabolome analyses revealed that commensal Lactobacillus suppressed colon tumorigenesis and progression through the modulation of gut microbiota and metabolites which included the down-regulation of secondary bile acids, sphingosine 1-phosphate (S1P) signaling in cancer and pyrimidine metabolism, as well as the production of anticarcinogenic compounds in tumor-bearing mice. Particularly, limitation the effects of S1P signaling via the modulation of sphingosine expression using probiotics, exerted antitumor efficiency.

Conclusions: The work indicated the feasibility of using an effectively safe strategy to prevent the occurrence of colon cancer, and provided a potential therapeutic target for new therapeutics strategy via altering S1P signaling in cancer.

\section{Background}

Colorectal cancer (CRC) remained the second leading cause of cancer death and almost 1.0 million cancer deaths occurred in $2020^{1}$. The polypoid adenomas in the colon and rectum were gradually developed CRCs through the multistep mechanism, including adenoma- carcinoma process ${ }^{2}$. Additionally, most of patients diagnosed at the middle and advanced stage owing to the longer development of malignancies in the colon and rectum and higher concealment than other cancers, which resulted in shorter treatment window and higher mortality rate ${ }^{3,4}$. Therefore, efficiently safe prevention strategies for colon cancers in early stages and the development of therapeutic target for new therapeutics strategy of colon cancer are priorities for cancer control.

One fifth of human malignancies are implicated in gut microbiota ${ }^{5}$. Accumulating evidence showed that gut microbiota was closely related to digestive diseases occurrence and health maintenance, especially colon cancers ${ }^{6,7}$.Therefore, the regulation of gut microbiota has been considered as a promising strategy for preventing and treating colon cancer ${ }^{8,9}$. Previously, phage-based intervention of gut microbiota and 
fecal microbiota transplant (FMT) that altered the gut microbiota of the patients and remodeled the homeostasis of intestinal microbiota was proved to be positively therapeutic effects ${ }^{10,11}$. However, the safety and operation standardization remained the biggest challenge for patients with colon cancer, which limited the development of cancer therapy based on the modulation of microbiota by phage-based intervention and FMT. Probiotics, as novel functional foods via microbiota-modulation to enhance host health and inhibit tumor carcinogenesis, have been attracted considerable interests. Most importantly, the recognized safety and complete standardization of probiotics were confirmed ${ }^{12}$. Recent studies revealed potentially positive effects of probiotics to colon cancer therapy ${ }^{13,14}$. Similarly, our previous reported results also showed that Lactobacillus casei inhibited significantly tumor progression in animal models of colon cancers ${ }^{15}$. However, the relationship between colon cancer and microbes is fairly complex. The mechanism in which microbes and the metabolites contribute to carcinogenesis, and the antitumor mechanisms influenced by microbiota and their metabolites with intervention of probiotic remain unclear.

Observation of the roles of microbes and the microbiota and its metabolites in colon cancer were of great importance for early prevention and treatment of colon cancers. Fecal microbial diversity is a useful tool for observation of the altered gut microbiome. Correspondingly, the intestinal metabolites, such as shortchain fatty acids (SCFAs), hydrogen sulphide, acetaldehyde, secondary bile acids, are involved in the initiation and/or progression of colorectal cancer ${ }^{16}$. Therefore, comprehensive microbiome and metabolomic analyses might provide an alternative approach to understanding colon cancer occurrence and development through associated alteration in the gut microbiota and their secreted metabolites with the intervention of probiotics.

The aim of this study was to investigate the preventive and therapeutic efficiency of commensal Lactobacillus, including JY300-8 and JMR-01, and how was involved to colon cancer occurrence and development for gut microbiome and its metabolites by the intervention of probiotics. Accordingly, the preventive and therapeutic efficiency (the tumor formation rate, the overall survival and tumor volume of tumor-bearing mice) in subcutaneous tumor models were assessed when oral administration for commensal Lactobacillus. Furthermore, the fecal microbiota composition and its metabolic profiles were performed to obtain evidence of antitumor effects of fecal microorganisms and metabolites. Our work provides an efficient prevention strategy for colon cancers via intervention of probiotic modulation, and also a potential therapeutic target for new therapeutics strategy of colon cancer.

\section{Materials And Methods}

\section{Cells and probiotic preparation}

The murine colon cancer cell lines CT-26 was purchased from Shanghai Cell Bank, cultured in $5 \% \mathrm{CO}_{2}$ of carbon dioxide incubator with RPMI 1640 containing $10 \%$ of fetal bovine serum and $1 \%$ of penicillin / streptomycin at $37^{\circ} \mathrm{C}$. 
Lactobacillus reuteri JMR-01 (NCBI Accession No. MT362007) was isolated from the feces of which cured breast cancer mice treated with carbon-ion beams. Lactobacillus casei JY300-8 ${ }^{15}$ was preserved in the Institute of Modern Physics, Chinese Academy of Sciences (IMP-CAS). JMR-01or JY300-8 was cultured statically at $37^{\circ} \mathrm{C}$ for $24 \mathrm{~h}$ in MRS broth, and its fermentation solution were centrifuged at 4000 $\mathrm{r} / \mathrm{min}$ for $10 \mathrm{~min}$ to acquire bacterial precipitates, respectively. Then bacterial precipitates of JY300-8 and JMR-01 were resuspended at $1 \times 10^{9} \mathrm{CFU} / \mathrm{mL}$ of concentration in PBS to obtain living bacteria JY300-8 and JMR-01, respectively. Subsequently, the living bacteria (LB) were prepared by the mixture of JY300-8 and JMR-01 at the ratio of $1: 2(\mathrm{v} / \mathrm{v})$; in addition, LB were autoclaved at $105^{\circ} \mathrm{C}$ for $30 \mathrm{~min}$ to prepared inactivated bacteria (IB).

\section{Antitumor efficacy of commensal Lactobacillus in subcutaneous colon cancer models}

\section{Experimental animal and subcutaneous colon cancer models}

BABL/C mice (Male, 6 weeks) were purchased from Lanzhou Veterinary Research Institute, Chinese Academy of Agricultural Sciences. Mice were maintained on sterilized mice chow, water at constant temperature $\left(20 \pm 3^{\circ} \mathrm{C}\right)$ and $40-70 \%$ of humidity conditions with a 12/12-h light/dark cycle in a specific pathogen-free facility after Institutional Animal Care and Use Committee approval at Biomedical Center, IMP-CAS. In accordance with the study schedule, the mice were sacrificed by euthanasia at the end of the experiment. All animal experiments complied with the ARRIVE guidelines and were carried out in accordance with the National Institutes of Health guide for the care and use of Laboratory animals (NIH Publications No. 8023, revised 1978).

A subcutaneous mouse model for colon cancer was developed using CT-26 cells. CT-26 cells were inoculated in RPMI 1640 medium containing $10 \% \mathrm{FBS}$ at $37^{\circ} \mathrm{C}$. At the exponential growth phase, $5 \times 10^{6}$ CT26 cells were suspended in $100 \mathrm{uL}$ cold PBS and subcutaneously injected into mice at right groin. Then tumor formation rate, tumor volume and survival rate were evaluated at alternate days. Tumor formation rate were counted by the ratio of the number of tumors forming mice to all mice. Tumor volume was $1 / 2 \times a^{2} b$, where $a$ and $b$ represented the largest and shortest tumor diameters, respectively.

\section{Experimental design and anti-tumor efficacy of commensal Lactobacillus}

Overall, the experimental scheme was composed of two processes, including the prevention and treatment of subcutaneous colon cancer model in mice. Throughout the experiment, the BABL/C mice were randomly divided into 3 groups with 30 animals each group, that was tumor control (CK), LB and IB group. In the prevention stage, the mice in control, LB and IB group, was performed semidiurnal gavage with $100 \mathrm{uL} /$ head of PBS, $0.1 \times 10^{9} \mathrm{CFU} /$ head of LB, $100 \mathrm{uL} /$ head of IB for 20 days, respectively. Subsequently, subcutaneous colon cancer models were induced by CT26 cells. Meanwhile, each group of mice were consistently performed prevention treatment as mentioned above, tumor formation and survival rate were evaluated when tumor model established successfully in control group (the mean tumor volume reached approximately $100 \mathrm{~mm}^{3}$ ). Then in the treatment stage of colon cancer, tumor- 
bearing mice were continued to oral administration of PBS, LB and IB until the end of experiment. Meanwhile, tumor volume and survival rate were evaluated at alternate days until the end of experiment when the highly significant different $(p<0.001)$ in mean volume between control group and treatment group were consistently achieved in three measurements. Meanwhile, the fecal samples were collected to analysis of the abundance of microbiota and its metabolome.

\section{Sample collection and detection based on the animal model}

For assessment of prevention effect of commensal Lactobacillus on colon cancer induced by CT26 cells in mice, survival and tumor formation rate in various groups were measured when tumor model established successfully in control. Furthermore, to evaluate the therapeutic efficacy of Lactobacillus on colon cancer, the tumor volume and survival were monitored in treatment stage. Finally, the fecal sample in various groups were aseptically collected for comparative analysis of the potentially altered microbiota and its metabolites in colon cancer mice by LB or IB intervention, then each of group was randomly chosen six of fecal samples to measure the microbiota and corresponding metabolites.

\section{The measurement of microbiome and metabolites and bioinformatic analysis}

\section{Microbiota sequencing of fecal samples}

Total genome DNA from fecal samples was extracted using TIANamp Bacteria DNA Kit (TIANGEN Biotechnology, China) according to the manufacturer protocol. Extracted DNA was further processed on an Illumina Miseq platform ${ }^{17}$. The V3-V4 region of the $16 \mathrm{~S}$ ribosomal RNA gene was targeted for amplicon sequencing using sequence-specific primers (341F: CCTAYGGGRBGCASCAG, 806R:

GGACTACNNGGGTATCTAAT) with attached Illumina adapter overhang sequences. All PCR reactions were carried out in $30 \mu \mathrm{L}$ reactions with $15 \mu \mathrm{L}$ of Phusion ${ }^{\circledR}$ High-Fidelity PCR Master Mix (New England Biolabs). Then PCR products was mixed in equidensity ratios and mixture PCR products was purified with AxyPrepDNA Gel Extraction Kit (AXYGEN). Subsequently, sequencing libraries were generated using NEB Next ${ }^{\circledR} U$ Ultra ${ }^{\text {TM }}$ DNA Library Prep Kit for Illumina (NEB, USA) following manufacturer's recommendations. The library quality was assessed on the Qubit@ 2.0 Fluorometer (Thermo Scientific) and Agilent Bioanalyzer 2100 system. At last, the library was sequenced on an Illumina Miseq platform and 250/300 bp paired-end reads were generated.

\section{Bioinformatic analysis of microbiota in fecal samples}

Sequence analysis was performed by UPARSE software package using the UPARSE-OTU and UPARSEOTUref algorithms. Sequences with $\geq 97 \%$ similarity were assigned to the same OTUs. Then OTU table and phylogenetic tree, UniFrac distance matrix were obtained and weighted ${ }^{18}$. The richness, inverse Simpson and Shannon index were estimated with the phyloseq (version 1.30.0) and vegan (version 2.5-6) packages in R. Graphical representation of the relative abundance of bacterial diversity from phylum to species were visualized using Krona chart. Cluster analysis was preceded by principal component analysis (PCA) using the QIIME software package ${ }^{19}$. LDA scores were calculated by LEfSe ${ }^{20}$ with the 
factorial Kruskal-Wallis test $(P<0.05)$ to confirm the discriminative genera between groups, and the logarithmic LDA threshold score was set at 2.0. Finally, the heatmap was drawn with heatmap package (version 1.10.12).

\section{Untargeted metabolomics of fecal samples using LC-MS/MS}

Quantitative analysis of untargeted metabolites was performed using Waters 2D UPLC (waters, USA) tandem Q Exactive high resolution mass spectrometer (Thermo Fisher Scientific, USA) ${ }^{21}$. Metabolites were extracted according to previously reported methods ${ }^{22}$. In short, $25 \mathrm{mg}$ fecal sample were weighed and extracted by directly adding $800 \mu \mathrm{L}$ of precooled extraction reagent (methanol: acetonitrile: water (2:2:1, v/v/v)), $10 \mu \mathrm{L}$ internal standards mix (L-Leucine-d3, L-PHENYLALANINE (13C9, 99\%), LTryptophan-d5, Progesterone-2,3,4-13C3) were added for quality. After homogenizing for $5 \mathrm{~min}$ using TissueLyser (JXFSTPRP, China) with adding two small steel balls, samples were performed ultrasound at $4^{\circ} \mathrm{C}$ for $10 \mathrm{~min}$ and incubated at $-20^{\circ} \mathrm{C}$ for $1 \mathrm{~h}$. And then centrifuged for $15 \mathrm{~min}$ at $25000 \mathrm{rpm}$ at $4{ }^{\circ} \mathrm{C}$, then the supernatant was performed vacuum drying. Subsequently, the metabolites were resuspended in 600 $\mu \mathrm{L}$ of $10 \%$ methanol and sonicated for $10 \mathrm{~min}$ at $4{ }^{\circ} \mathrm{C}$, then the supernatants were used for LC-MS analysis after centrifuging for $15 \mathrm{~min}$ at $25000 \mathrm{rpm}$ at $4^{\circ} \mathrm{C}$. Additionally, $50 \mu \mathrm{L}$ of the supernatant of each sample and mixed it into a QC quality control sample to evaluate the repeatability and stability of the LCMS analysis process.

\section{The analysis of metabolomics data}

LC-MS/MS data were analyzed by Compound Discoverer 3.1 (Thermo Fisher Scientific, USA) software. And the metabolome R software package metaX were employed to perform data preprocessing, statistical analysis, metabolite classification and functional annotation ${ }^{23}$. Metabolites were identified by combining of mzCloud, the Human Metabolome Database (HMDB), Kyoto Encyclopedia of Genes and Genomes (KEGG) and Lipid Maps databases. Subsequently, principal component analysis (PCA) was employed to analyze similarities and differences within groups as well as outliers ${ }^{24}$. Next, the significant differential metabolites were screened by variable important for the projection (VIP) calculated using partial least squares discriminant analysis (PLS-DA ${ }^{25}$, combined with fold change (FC) of a single variable analysis and $\mathrm{p}$ value of Student's $\mathrm{t}$ test $^{26}$.

\section{Coalition analysis of 16S rDNA amplicon sequencing and untargeted metabolomics}

Spearman statistical method was employed to analyze the correlation coefficient between the significant difference microflora and significant difference metabolites, and then $\mathrm{R}$ and Cytoscape software were used to perform the analysis of the matrix heat map, hierarchical clustering, correlation network to investigate the interaction relationship between microbiota and metabolites.

\section{Statistical analysis}


Statistical analysis was performed using SPSS 22.0 software (SPSS Inc., Chicago, IL, USA) and plotted using Origin 2019 (OriginLab Corp., Northampton, MA, USA) and GraphPad Prism 8.0.1 software (GraphPad Inc., California, CA, USA). The significant differences between the two groups were analyzed using an independent samples T-test using by one-way ANOVA with Tukey-Kramer comparison test. NS, not significant, ${ }^{*} P<0.05,{ }^{\star *} \mathrm{P}<0.01,{ }^{\star \star *} \mathrm{P}<0.001$ indicated statistical significance level.

\section{Results}

\section{Antitumor efficacy of commensal Lactobacillus in subcutaneous colon cancer models in mice}

To determine whether commensal Lactobacillus exerted prevention and treatment activity for colon cancer in vivo, we assessed its anti-tumor efficacy using the CT26 cells induced-mouse colon cancer model. The experimental scheme for tumor prevention and treatment by oral administration of commensal Lactobacillus were shown as Fig.1a, and the anti-tumor efficacy of commensal Lactobacillus on CT26 subcutaneous colon cancer in mice were presented as Fig1(b-e). As shown in Fig1b, in the prevention stage of colon cancer induced by CT26 cells, the survival rates of mice were $100 \%$ in tumor control, LB and IB group for 20 days oral administration for probiotics, showing that probiotics were safety for animal. Additionally, the tumor formation rates were $13.33 \%$ and $16.67 \%$ in LB and IB group, while that was reached $96.67 \%$ in control after 10 days CT26 cells were subcutaneously injected, which indicated that living and inactivated bacteria treated-mice reduced significantly the tumor formation rate compared to the tumor controls $(\mathrm{p}<0.01)$.

Moreover, in the treatment stage of colon cancer induced by CT26 cells, tumor growth was much faster in the tumor control group than in those treated with living bacteria and inactivated bacteria treated group ( $p$ $\nabla 0.001$ ) (Fig.1d), and the mean tumor volume was $4920.63 \pm 462.4 \mathrm{~mm}^{3}$ in tumor control group, while they were $1712.45 \pm 388.95 \mathrm{~mm}^{3}$ and $1909.95 \pm 292.9 \mathrm{~mm}^{3}$ in living bacteria and inactivated bacteria treatment groups at the end of the experiment, respectively. In addition, though the tumor new formation rates were gradually increased in LB and IB group, which was $66.67 \%$ and $73.33 \%$ of tumor formation rates, those were remarkable lower than control group $(p<0.05)$, which was an indirectly proof that oral administration for living commensal Lactobacillus could suppressed the colon cancer progression, followed by inactivated bacteria treated-group. Meanwhile, the survival ratios of bearing mice were $93.33 \%$ both in tumor control group and IB group, when compared to $100 \%$ of survival rate in LB group at 30 days. Therefore, these results demonstrated that the commensal Lactobacillus reduced significantly the occurrence of colon cancer, suppressed sufficiently the tumor growth and progression, and enhanced the survival rate of bearing tumor mice.

\section{Commensal Lactobacillus enhanced the antitumor responsiveness by modulating gut microbiota}

In order to further explore the potential effects of the commensal Lactobacillus on gut microbiota in the CT26 induced-murine model of colon cancer, and assess the interaction of altered microbiota and colon cancer progression, Miseq sequencing analysis of the V3-V4 region of the 16S ribosomal RNA gene of the 
fecal samples were performed. The structure and abundance of microbiota in three groups were shown in Fig.2. According to the rarefaction curve (Fig.2a), the amount of sequencing data in samples was sufficient to perform the analysis of the diversity and abundance of gut microbiota. At the end of the experiment, the diversity of microbial flora (Shannon index and Simpson index) was no significant difference among the tumor control (CK), living bacteria, and inactivated bacteria group (Fig.2b). The abundance of microbial flora both in living bacteria and inactivated bacteria were higher than that of tumor control group $(\mathrm{p}<0.001)$, and there was not significant difference in living bacteria and inactivated bacteria (Fig.2b). Additionally, Principle-coordinates analysis ( $P C \circ A)$ results showed that phylogenetic community structures were notably different between tumor control group and others, however the PCoA plots also suggested that the living bacteria and inactivated bacteria groups were not obviously dispersed at 30 days (Fig. 2c). It demonstrated that commensal Lactobacillus administration significantly changed the gut microbiota composition of tumor-bearing mice. As the similarity of results in Fig 2b, Venn analysis also showed that living bacteria treatment affected significantly the structure of the intestinal flora (Fig.2d). Distribution and abundance of microbial taxa at the phylum level in three groups at 30 days were examined as shown in Fig. 2e. The results indicated that bacteria in the Bacteroidetes and Firmicutes phyla dominated the mouse gut microbiota. The dominant phyla in the gut microbiota were Bacteroidetes, Firmicutes, Proteobacteria and TM7 (relative abundance $>0.5 \%$ ). At genus level, the fluctuation of relative abundance depended on the various treatment. The dominant gut microbiota were Bacteroides, Oscillospira, Prevotella, Ruminococcus, AF12, Roseburia, Parabacteroides, Coprococcus, Ruminococcus (relative abundance $>0.5 \%$ ) (Fig. 2f.). Compared to the control, the living bacteria treated group was significantly increased in Oscillospira, Prevotella, AF12, Roseburia, Coprococcus, while Bacteroides, Ruminococcus and Parabacteroides increased. These results showed that the gut microbiota with the capacity of producing short chain fatty acid (SCFAs) were enriched in living bacteria treatment group. Similarly, the Prevotella, AF12, Roseburia, Coprococcus and Ruminococcus were increased, while the Bacteroides and Ruminococcus reduced in inactivated bacteria treatment group in comparison to tumor control group. Thus, our data from colon cancer mouse with various treatment demonstrated that both the living bacteria and inactivated bacteria contributed to alleviating dysbiosis induced by tumor by modulating gut microbiota structure with respect to increased alpha and beta diversity and the proportion of potentially beneficial taxa, which enhanced the anti-tumor response.

Furthermore, in order to determine which bacteria were most likely associated with cancer prevention and treatment efficiency between LB and IB group, we employed the linear discriminant analysis (LDA) and effect size (LEfSe) methods to calculate the LDA scores for the three samples at 30 days. The lists of taxonomic clades ranked according to the effect size, which were differential among groups with statistical and biological significance were shown in Fig. $2 \mathrm{~g}$. The results indicated that, between the living bacteria and control groups, Coprococcus, AF12, Lactobacillus, Enterobacter and Turicibacter were most discriminative at genus level in living bacteria group. Similarly, Coprococcus was most differential in inactivated bacteria group. However, Butyricimonas, Sphingomonas and Prevotella were most discriminative in tumor control group. Between living bacteria and inactivated bacteria group, most discriminative in living bacteria group included, Lactobacillus and Escherichia. 


\section{The analysis of untargeted metabolites associated with anti-tumor efficiency}

The quantitative metabolomics technology was used to obtain the complete metabolic profiles of gut microbiota in colon cancer mice with various treatments. Overall, As shown in Fig. S1a-b (Additional file 1: Fig. S1a-b), PCA analysis in QC samples of positive and negative ion model showed that the data collection process was reliable. At the same time, both positive and negative ion model, control group and LB group, and control group and IB group were highly distinguished. These results indicated significant differences in metabolic profiles with LB and IB treatment when compared with control. It was also indirectly proved that living bacteria or inactivated bacteria had a great influence on the metabolism of microbiota in colon cancer mouse model. However, the fecal metabolic profiles with LB and IB treatment were not obviously distinguished, which was indicated that there were similar metabolic profiles each other.

Furthermore, the metabolites with significant difference between various treatments were obtained (Additional file 2: Table. S1-S3). To further screen the highly significant difference metabolites, more stricter screening conditions were employed from significant difference metabolites (VIP $>3, F C>1$ or FC $<0.83$ as well as $p<0.01$ ). Accordingly, cluster analysis of differential metabolites for all samples were shown as Fig.3. Compared to control, the up-regulated significant different metabolites in the LB groups were 9-oxooDE ( $\left.p=4.45 \times 10^{-5}, \mathrm{VIP}=10.94\right), 9(S)$-HODE $(p=0.00096, \mathrm{VIP}=17.20)$, succinic acid $(p=0.015$, $\mathrm{VIP}=3.455)$. However, linoleic acid $(P=1.04 \times 10-5, \mathrm{VIP}=3.45)$, pantothenate $(\mathrm{p}=0.00016, \mathrm{VIP}=3.237)$, cholic acid $(p=0.006, \mathrm{VIP}=3.361)$, chenodeoxycholate $(\mathrm{p}=2.92 \times 10-5, \mathrm{VIP}=11.946)$, deoxycholic acid $\left(p=9.408 \times 10^{-6}, V I P=21.118\right), 4$-guanidinobutyric acid $(p=0.0021, V I P=2.665)$, sphingosine $(p=0.01$, $\mathrm{VIP}=4.366)$ were main the down-regulated significant different metabolites in colon cancer mice administrated living bacteria, respectively. Moreover, the significant different metabolites, which included urea $(p=0.0003, V I P=5.681)$, creatinine $(p=0.0024, V I P=11.401)$, pantothenate $(p=0.00019, V I P=3.006), 1$ Methylnicotinamide $(p=0.0085, \mathrm{VIP}=6.287)$, cholic acid $(p=0.0085, \mathrm{VIP}=10.833)$, chenodeoxycholate $(p=0.000027, V I P=12.171)$, deoxycholic acid $\left(p=9.56 \times 10^{-6}, V I P=21.723\right)$, thymine $(p=0.000046$, VIP=3.745), uracil $(p=0.00277, V I P=5.098)$, cytosine $(p=0.0036, V I P=5.985)$, linoleic acid $\left(p=5.703 \times 10^{-6}\right.$, $\mathrm{VIP}=3.212)$, anthranilic acid $(\mathrm{p}=0.0066, \mathrm{VIP}=8.311)$, sphingosine $(\mathrm{p}=0.0063, \mathrm{VIP}=4.96)$, were lower expression level in IB group than tumor control group. In contrast, the up-regulated significant different metabolites in the IB groups were maltotriose $\left(p=1.65 \times 10^{-6}, V I P=3.133\right), D-(+)-$ Melibiose $(p=0.0022$, VIP=3.76), Alpha-D-Glucose $(p=0.0022, V I P=6.911)$ compared to tumor control. In comparison to IB group, the different metabolites that were significantly up-regulated in the LB group were succinate $(p=0.0056$, VIP = 4.285); and the metabolites, including N-Acetyl-D -glucosamine $(p=0.0015, \mathrm{VIP}=3.050)$, cytidine ( $p$ $=0.010, \mathrm{VIP}=1.714$ ), were down-regulated.

Additionally, the results of KEGG enrichment analysis indicated that significant different metabolites that were likely associated with the colon cancer progression, were enriched in Bile secretion, Central carbon metabolism in cancer, Phenylalanine metabolism and Alanine, Arginine and proline metabolism as well as Apoptosis process in control_LB groups, respectively (Additional file 3: Fig. S1). In the control_IB 
groups, the significant different metabolites were enriched in Bile secretion, Pyrimidine metabolism, Apoptosis, Arginine and proline metabolism, respectively (Additional file 4: Fig. S1). While the significant different metabolites in the LB_IB groups were enriched in Pyrimidine metabolism (Additional file 5: Fig. S1).

\section{Coalition analysis of microbiome and metabolomics}

In order to obtain a global understanding of interaction with microbiota and its metabolites in colon cancer progression via different treatments, a coalition analysis of the $16 \mathrm{~S}$ microbial diversity and metabolomics were performed. As spearman correlation analysis on significant difference microbiota and metabolites in control_LB groups, sphingosine that involved in Apoptosis was significantly positively correlated with Veillonella, and negatively correlated with Coprococcus and Prevotella. And cholic acid and chenodeoxycholate was negatively correlated with Dehalobacterium, respectively. Moreover, deoxycholic acid was positively correlated with Porphyromonas. And succinate was positively correlated with Coprococcus and Dehalobacterium. (Fig4.a, b). Similarly, the coalition analysis of significant difference microbiota and metabolites in the control_IB groups, showing that uracil, thymine and cytosine, the key metabolites involved in Pyrimidine metabolism signaling pathway, had the same expression pattern, which were positively correlated with Prevotella, negatively correlated with Bacteroides and Flexispira, respectively. Cholic acid, chenodeoxycholate and deoxycholic acid showed similar expression patterns, and were positively correlated with Prevotella, Flexispira, Bacteroides in addition to Porphyromonas with deoxycholic acid. Additionally, sphingosine was positively correlated with Flexispira, and negatively correlated with Prevotella (Fig 4.c, d).

\section{Discussion}

Recently, the relationship among an altered gut microbiota and its metabolites with colon cancer provides a potential strategy for prevention and treatment of colon cancer via the modulation of gut microbiota using probiotics ${ }^{27,28}$. Probiotics are the most commonly consumed food supplements owing to recognized safety and complete standardization ${ }^{12}$, thus probiotics are consider as most potential safe agents for cancer prevention in the form of food supplements, especially colon cancer. Accumulating evidence showed that probiotics, such as Lactobacillus supplementation, could prevent the animal that were susceptible to colon cancer against carcinoma effects in vivo as shown in Table 1. According to Table 1, many probiotics might exert protective effects against colon cancer, but did not act on preexisting colon cancer. However, our results demonstrated that L.casei JY300-8 and L.reuteri JMR-01 pretreated-group not only decreased significantly the tumor formation rate but also suppressed remarkably the tumor growth and enhanced the survival compared to those of in tumor control. Similarly, our previous studies also verified that $L$. casei JY300-8 inhibited significantly tumor progression and stronger inhibitory effect on tumor (83.48\%) in comparison to the therapy drug (DFUR) for colon cancer $(65.65 \%)$ in animal models of colon cancers ${ }^{15}$. Additionally, although the highest protective effects were reached via probiotic VSL\#3 - treated animals (there were no animals developed carcinoma), the merely $29 \%$ of animal developed carcinoma in control group ${ }^{29}$. Accordingly, oral administration of JY300-8 and 
JMR-01 presented more potential protective effects against the occurrence of colon cancer induced by colon cancer cells, which were considered as more promising probiotics in colon cancer prevention and anti-cancer therapies.

\section{Table 1 Effects of probiotics on colon cancer in animal models}

\begin{tabular}{|c|c|c|c|}
\hline Strains & $\begin{array}{l}\text { Colon cancer } \\
\text { model }\end{array}$ & Antitumor efficiency & Reference \\
\hline VSL\#3 & $\begin{array}{l}\text { Colitis-associated } \\
\text { colorectal cancer } \\
\text { in rats }\end{array}$ & 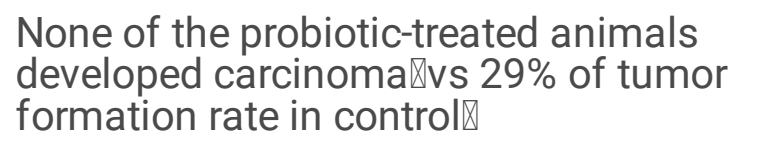 & 29 \\
\hline $\begin{array}{l}\text { Heat-killed cells of } E \text {. } \\
\text { faecalis }\end{array}$ & $\begin{array}{l}\text { DSS-induced } \\
\text { colitis in mice }\end{array}$ & $\begin{array}{l}\text { E. faecalis may exert protective effects } \\
\text { against CRC but does not act on pre- } \\
\text { existing CRC. }\end{array}$ & 30 \\
\hline $\begin{array}{l}\text { Lactobacillus } \\
\text { plantarum }\end{array}$ & $\begin{array}{l}\text { DSS-induced in } \\
\text { mice }\end{array}$ & Reduction the tumor incidence & 31 \\
\hline $\begin{array}{l}\text { Bacillus } \\
\text { polyfermenticus }\end{array}$ & $\begin{array}{l}\text { DLD- } 1 \text { cells } \\
\text { injection in mice }\end{array}$ & Reduction the tumor incidence & 32 \\
\hline $\begin{array}{l}\text { L. acidophilus, L. casei, } \\
\text { L. lactis biovar } \\
\text { diacetylactis DRC-1 }\end{array}$ & $\begin{array}{l}\text { DMH induced in } \\
\text { rat }\end{array}$ & $\begin{array}{l}\text { Reduction the tumor incidence and } \\
\text { multiplicity }\end{array}$ & 33 \\
\hline L. plantarum YYC-3 & $\begin{array}{l}\text { In the } A P C^{M i n /+} \\
\text { mouse model of } \\
\text { colon cancer }\end{array}$ & $\begin{array}{l}\text { Tumor incidence was } 22.22 \% \text { (VS } 88.33 \\
\% \text { in control) }\end{array}$ & 34 \\
\hline JY300-8 and JMR-01 & $\begin{array}{l}\text { CT26 cells } \\
\text { injection in mice }\end{array}$ & $\begin{array}{l}\text { Reduction the tumor incidence }(13.33 \% \\
\text { vs } 96.67 \% \text { in control) }\end{array}$ & $\begin{array}{l}\text { In this } \\
\text { study }\end{array}$ \\
\hline
\end{tabular}

Probiotics could regulate gut microbiota structure and composition, thus modulating occurrence and development of colon cancer ${ }^{35,36}$. The present studies suggested that the structure and abundance of gut microbiota were correlated with the development of colon cancer via administration for living or inactivated probiotics. And the major microbial alterations correlated with colon cancer progression were shown in Fig.5. The Flexispira, Bacterodes, Porphyromonas, Clostridium and Escherichia enriched in tumor control, especially Clostridium and Escherichia were extensively existed in all tumor mice, were possibly played the important role in the development and progression of colon cancer. Similarly, recent research suggested that Fusobacterium nucleatum, Escherichia coli, Bacteroides fragilis were promoted the neoplastic processes in epithelial cells ${ }^{37}$. We speculated that some species might be associated with colon cancer, including Flexispira and Porphyromonas, which possibly secreted the harmful metabolites, such as cholic acid, chenodeoxycholate, deoxycholic acid. Additionally, the Coprococcus, Veillonella, Lactobacillus, Bifidobacterium and Dehalobacterium in oral administration of living JY300-8 and JMR-01 were likely reduced the occurrence and development of colon cancer. As previously evidence suggested that Coprococcus, produced beneficial metabolites, such as short chain fatty acids including acetate, 
propionate, butyrate, exerted the antitumor efficiency ${ }^{38}$. Meanwhile, Veillonella could fermented lactate produced by Lactobacillus to yield the acetate, propionate ${ }^{39}$. However, the prevotella that associated with gut inflammation ${ }^{40}$, was enriched in administration for inactivated JY300-8 and JMR-01. Fortunately, an overgrowth of Prevotella was correlated with a reduction of Lactobacillus $^{41}$, thus oral administration living JY300-8 and JMR-01 in IB group would be mitigated the negatively effects of Prevotella. Overall, the altered microbiota was associated with the colon cancer. The Flexispira, Bacterodes, Porphyromonas, Clostridium and Escherichia might play an important role in progression of colon cancer. Contrarily, the Coprococcus, Veillonella, Lactobacillus, Bifidobacterium and Dehalobacterium were possibly exerted the protective effects on colon cancer susceptible mice.

Microbial metabolites in the gut have a particularly important role in progression of colon cancer ${ }^{16}$. Recent evidence suggested that the bile acid, especially deoxycholic acid, has been implicated in carcinogenesis of intestine owing to the generation of ROS and reactive nitrogen species (RNS), both of which cause DNA damage ${ }^{42,43}$. In the present study, bile acids including cholic acid, deoxycholic acid, existed extensively in intestine of tumor control, while the concentration of bile acids were significantly decreased when oral administration of living JY300-8 and JMR-01 or inactivated JY300-8 and JMR-01, which were showed that the increased fecal bile acid concentrations were positively correlated with the incidence and progression of colon cancer. In addition, the concentration of sphingosine was significantly up-regulation in colon cancer tumor, while the sphingosine was remarkably decreased via the intervention of living probiotics or inactivated probiotics. Sphingosine-1-phosphate(S1P), is formed by the phosphorylation of sphingosine via the sphingosine kinase1(SK1) and/or sphingosine kinase2(SK2) in vivo, promotes tumorigenesis, transformation (transformation the normal cells to cancerous cells), neovascularization (provides cancer cells with nutrients and oxygen), tumor cell growth and survival ${ }^{44}$. Moreover, the reported research demonstrated that higher levels of sphingosine was used as selective pressure to increase SK1 expression and thereby converting sphingosine to S1P to promote tumor cell survival ${ }^{45}$. Therefore, strategies that S1P pathway therapeutics for cancer have been adopted to limit the effects of S1P signaling in cancer, including the reduction of released S1P46, inhibition of SK1 and/or SK2 ${ }^{47}$ and targeting of specific S1P receptors ${ }^{48}$. Importantly, in our study, the concentration of sphingosine in the intestine of colon cancer mice was significantly regulated by oral administration living or inactivated bacteria (JY300-8 and JMR-01), and the concentration reduction of S1P that transformed from sphingosine in intestine or other organs, thus exerting antitumor efficiency, including reduction of transformation the normal cells to cancerous cells, neovascularization, promotion the apoptosis of tumor cell. Additionally, the sphingosine was a strongly positive correlation with Veillonella and Flexispira, and negatively correlated with Coprococcus and Prevotella. Notably, the expression level of sphingosine might be regulated in colon cancer via intervention of excellent probiotics, which provided a potential therapeutic target for new therapeutics strategy via altering S1P signaling and function in cancer.

Additionally, other microbial metabolites in oral administration of living bacteria(JY300-8 and JMR-01), such as succinic acid, which was fermented to produce propionate via the succinate pathway in the context of microbiome ${ }^{38}, 9-0 x 0 O D E^{49}$, exerted anticarcinogenic activity. As the similar report that 
conjugated linoleic acids, such as 9-OxoODE, were produced from linoleic acid by the fermentation of Lactobacillus and Bifidobacterium in the intestinal lumen, thus exerting its beneficial effects ${ }^{50}$. Moreover, pyrimidine metabolism, including thymine, cytosine and uracil, is a critical pathway for DNA replication, RNA synthesis. Hence, increased pyrimidine metabolism could guarantee uncontrolled growth of tumors ${ }^{51}$. In the present study, we demonstrated that the concentrations of thymine, uracil and cytosine implicated in pyrimidine metabolism pathway were depleted when administrated the inactivated bacteria JY300-8 and JMR-01 for colon cancer. Consistent with our observation, changes in pyrimidine metabolism regulated cancer cell proliferation ${ }^{52}$. Furthermore, the thymine, cytosine and uracil had similar expression patterns, all of these were positively correlated with Flexispira, Bacteroides, Porphyromonas and negatively correlated with Prevotella. Accordingly, this link between pyrimidine metabolism and tumorigenesis might provide novel targets for anticancer therapy via modulation of microbiota and its bioactive metabolites using excellent probiotics.

Interestingly, based on the shifts of the microbiota and their excreted bioactive metabolites in colon cancer with various treatments, we found that these were complementary in the process of colon cancer prevention and therapy by intervention of living bacteria (JY300-8 and JMR-01) and inactivated bacteria (JY300-8 and JMR-01). The common points of them were that they both reduced the concentration of the bile acids and sphingosine to attenuate the cell DNA damage and suppress the tumor tumorigenesis, transformation, neovascularization, growth and survival. The differences were remarkable, another antitumor efficiency of oral administration for living bacteria was production of compounds with anticarcinogenic activity, such as short-chain fatty acids and conjugated linoleic acid(9-OxoODE). While the decreased pyrimidine metabolism level, including thymine, cytosine and uracil, were another important anti-cancer cell proliferation factor when administrated of inactivated bacteria. Meanwhile, the enrichment of Prevotella and depletion of Lactobacillus, Bifidobacterium and Coprococcus were likely detrimental to their healthy and antitumor efficiency, in agreement with the fact that the overall survival rate of mice in inactivated bacteria treatment was reduced in comparison to those of treated with living bacteria. To further enhance the antitumor efficiency, combination living bacteria (JY300-8 and JMR-01) with inactivated bacteria (JY300-8 and JMR-01) might be potentially strategy used as precision cancer therapeutics owing to the coordination and complementarity of their antitumor activity, which were needed to further studies to validate our hypothesis in preclinical colon cancer model and in patient with colon cancer.

\section{Conclusions}

In conclusion, our data demonstrated that commensal Lactobacillus suppressed colon tumorigenesis and progression through the modulation of gut microbial homeostasis and metabolites. For tumorbearing mice with oral administration of living JY300-8 and JMR-01, the therapeutic efficiency to colon cancer mice were performed by down-regulation of secondary bile acids and S1P signaling, and the production of anticarcinogenic compounds. Similarly, the results revealed that reduction of secondary bile acids, S1P signaling in cancer and pyrimidine metabolism pathway could exert antitumor function 
when orally administrated inactivated JY300-8 and JMR-01 for tumor-bearing mice. Therefore, modulation of gut microbiota and metabolites by intervention of probiotics could be a potential therapeutic strategy in the prevention and treatment of colon cancer.

\section{Abbreviations}

S1P: Sphingosine 1-Phosphate; CRC: Colorectal cancer; FMT: Fecal microbiota transplant; SCFAs: Shortchain fatty acids; IMP-CAS: Institute of Modern Physics, Chinese Academy of Sciences; LB: Living bacteria; IB: Inactivated bacteria; CK: Tumor control; OUT: Operational taxonomic unit; PCA: Principal component analysis; KEGG: Kyoto Encyclopedia of Genes and Genomes; HMDB: Human Metabolome Database; VIP: Variable important for the projection; PLS-DA: Partial least squares discriminant analysis; FC: Fold change; LDA: linear discriminant analysis; LEfSe: linear discriminant analysis and effect size. SK1: Sphingosine kinase1; SK2: Sphingosine kinase2.

\section{Declarations}

\section{Acknowledgments}

We thank the Biomedical Central of Institute of Modern Physics, Chinese Academy of Sciences for providing all necessary equipment. And we are especially grateful to Prof Qiang Li and Dr Ting Zhao for providing guidance and support on this research.

\section{Author contributions}

Fuqiang Xu: Investigation, Writing Original draft, Review \& Editing. Qiaoqiao Li: Data curation and Investigation, Formal analysis. Shuyang Wang: Conceptualization, Supervision, Review \& Editing. Miaoyin Dong: Validation and Formal analysis. Guoqing Xiao: Conceptualization, Supervision and Funding acquisition. Jin Bai: Review \& Editing. Junkai Wang: Review and Revision. Xisi Sun: Revision and Formal analysis. All authors read and approved the final manuscript.

\section{Funding}

This work was supported by the "HONGGUANG" Special Project of Chinese Academy of Sciences (No. KFJ-HGZX-001), National Natural Science Foundation of China (No. 12075294), Key Research and Development Programs of Gansu Province (21YF5FA138), the Cooperation Project of Gansu Academy of Sciences (2020HZ-01), the Local Cooperation Project of the Chinese Academy of Sciences (YD-2021-01), the Project of Agricultural Science and Technology of Yunnan Province (202101AE090028).

\section{Availability of data and materials}

16S rDNA in this study have been submitted in the NCBI database, and we obtained BioProject accession number SUB11052277. 


\section{Ethics approval and consent to participate}

All animal experiments complied with the ARRIVE guidelines and were carried out in accordance with the National Institutes of Health guide for the care and use of Laboratory animals (NIH Publications No. 8023, revised 1978).

\section{Consent for publication}

Not applicable.

\section{Competing interests}

The authors declare no competing interests.

\section{Author details}

${ }^{1}$ Institute of Modern Physics, Chinese Academy of Sciences, Lanzhou, Gansu, PR China

${ }^{2}$ College of Nuclear Science and Technology, University of Chinese Academy of Sciences, Beijing, PR China

${ }^{3}$ Institute of Biology, Gansu Academy of Sciences, Lanzhou, Gansu, PR China

${ }^{4}$ College of Physics, Northwest Normal University, Lanzhou, Gansu 730000, PR China

*Correspondence: Prof Shuyang Wang (wangsy@impcas.ac.cn). Institute of Modern Physics, Chinese Academy of Sciences, 509 Nanchang Rd., Lanzhou, Gansu 730000, PR China. Prof Guoqing Xiao (xiaogq@impcas.ac.cn). Institute of Modern Physics, Chinese Academy of Sciences, 509 Nanchang Rd., Lanzhou, Gansu 730000, PR China.

${ }^{5}$ These authors contributed equally.

\section{References}

1 Sung, H. et al. Global cancer statistics 2020: GLOBOCAN estimates of incidence and mortality worldwide for 36 cancers in 185 countries. CA Cancer J Clin, doi:10.3322/caac.21660 (2021).

2 Fearon $\rrbracket$, E. R. \& Vogelstein, B. A Genetic Model for Colorectal Tumorigenesis. Cell 61, 759-767, doi:10.1016/0092-8674(90)90186-I (1990).

3 Peuker, K. et al. Epithelial calcineurin controls microbiota-dependent intestinal tumor development. Nat Med 22, 506-515, doi:10.1038/nm.4072 (2016).

4 Sia^n Jones, W.-d. C., Giovanni Parmigiani, Frank Diehl, Niko Beerenwinkel, , T. A., Arne Traulsen, M. A. N., Christopher Siegel, Victor E. Velculescu, Kenneth W. Kinzler, \& Bert Vogelstein, J. W., and Sanford D. 
Markowitz. Comparative lesion sequencing provides insights into tumor evolution. PNAS 105, 42834288 (2008).

5 de Martel, C. et al. Global burden of cancers attributable to infections in 2008: a review and synthetic analysis. The Lancet Oncology 13, 607-615, doi:10.1016/s1470-2045(12)70137-7 (2012).

6 Richard, M. L. \& Sokol, H. The gut mycobiota: insights into analysis, environmental interactions and role in gastrointestinal diseases. Nat Rev Gastroenterol Hepatol 16, 331-345, doi:10.1038/s41575-019-0121-2 (2019).

7 Tremaroli, V. \& Backhed, F. Functional interactions between the gut microbiota and host metabolism. Nature 489, 242-249, doi:10.1038/nature11552 (2012).

8 Helmink, B. A., Khan, M. A. W., Hermann, A., Gopalakrishnan, V. \& Wargo, J. A. The microbiome, cancer, and cancer therapy. Nat Med 25, 377-388, doi:10.1038/s41591-019-0377-7 (2019).

9 Zagato, E. et al. Endogenous murine microbiota member Faecalibaculum rodentium and its human homologue protect from intestinal tumour growth. Nat Microbio/ 5, 511-524, doi:10.1038/s41564-0190649-5 (2020).

10 Zheng, D. W. et al. Phage-guided modulation of the gut microbiota of mouse models of colorectal cancer augments their responses to chemotherapy. Nat Biomed Eng 3, 717-728, doi:10.1038/s41551-0190423-2 (2019).

11 Kelly, C. P. Fecal microbiota transplantation-an old therapy comes of age. N Engl J Med 368, 474-475, doi:10.1056/NEJMe1214816 (2013).

12 O'Toole, P. W., Marchesi, J. R. \& Hill, C. Next-generation probiotics: the spectrum from probiotics to live biotherapeutics. Nat Microbio/ 2, 17057, doi:10.1038/nmicrobiol.2017.57 (2017).

13 Zhu, Y., Michelle Luo, T., Jobin, C. \& Young, H. A. Gut microbiota and probiotics in colon tumorigenesis. Cancer Lett 309, 119-127, doi:10.1016/j.canlet.2011.06.004 (2011).

14 Appleyard, C. B. et al. Pretreatment with the probiotic VSL\#3 delays transition from inflammation to dysplasia in a rat model of colitis-associated cancer. Am J Physiol Gastrointest Liver Physio/ 301, G10041013, doi:10.1152/ajpgi.00167.2011 (2011).

$15 \mathrm{Xu}$, F. et al. Lactobacillus casei JY300-8 generated by 12C6+ beams mutagenesis inhibits tumor progression by modulating the gut microbiota in mice. Journal of Functional Foods $\mathbf{8 7}$, doi:10.1016/j.jff.2021.104779 (2021).

16 Louis, P., Hold, G. L. \& Flint, H. J. The gut microbiota, bacterial metabolites and colorectal cancer. Nat Rev Microbio/ 12, 661-672, doi:10.1038/nrmicro3344 (2014). 
17 Lundberg, D. S., Yourstone, S., Mieczkowski, P., Jones, C. D. \& Dangl, J. L. Practical innovations for high-throughput amplicon sequencing. Nat Methods 10, 999-1002, doi:10.1038/nmeth.2634 (2013).

18 Callahan, B. J. et al. DADA2: High-resolution sample inference from Illumina amplicon data. Nat Methods 13, 581-583, doi:10.1038/nmeth.3869 (2016).

19 Bolyen, E. et al. Reproducible, interactive, scalable and extensible microbiome data science using QIIME 2. Nat Biotechno/ 37, 852-857, doi:10.1038/s41587-019-0209-9 (2019).

20 Segata, N. et al. Metagenomic biomarker discovery and explanation.pdf. Genome Biology 12(6):R60, doi:10.1186/gb-2011-12-6-r60 (2011).

21 Benton, H. P. et al. Autonomous metabolomics for rapid metabolite identification in global profiling. Anal Chem 87, 884-891, doi:10.1021/ac5025649 (2015).

22 Dunn, W. B. et al. Procedures for large-scale metabolic profiling of serum and plasma using gas chromatography and liquid chromatography coupled to mass spectrometry. Nature Protocols 61060 1083, doi:10.1038/nprot.2011.335 (2011).

23 Wen, B., Mei, Z., Zeng, C. \& Liu, S. metaX: a flexible and comprehensive software for processing metabolomics data. BMC Bioinformatics 18, 183, doi:10.1186/s12859-017-1579-y (2017).

24 Barker, M. \& Rayens, W. Partial least squares for discrimination. Journal of Chemometrics 17, 166-173, doi:10.1002/cem.785 (2003).

25 Westerhuis, J. A. et al. Assessment of PLSDA cross validation. Metabolomics 4, 81-89, doi:10.1007/s11306-007-0099-6 (2008).

26 Kuhl, C., Tautenhahn, R., Bottcher, C., Larson, T. R. \& Neumann, S. CAMERA: an integrated strategy for compound spectra extraction and annotation of liquid chromatography/mass spectrometry data sets. Anal Chem 84, 283-289, doi:10.1021/ac202450g (2012).

27 Zheng, D. W. et al. Prebiotics-Encapsulated Probiotic Spores Regulate Gut Microbiota and Suppress Colon Cancer. Adv Mater 32, e2004529, doi:10.1002/adma.202004529 (2020).

28 Song, M., Chan, A. T. \& Sun, J. Influence of the Gut Microbiome, Diet, and Environment on Risk of Colorectal Cancer. Gastroenterology 158, 322-340, doi:10.1053/j.gastro.2019.06.048 (2020).

29 Caroline B. Appleyard, M. L. C., Angel A. Isidro, Janelle C. Arthur, Christian Jobin, and Claudio De Simone. Pretreatment with the probiotic VSL\#3 delays transition from inflammation to dysplasia in a rat model of colitis-associated cancer. Am J Physiol Gastrointest Liver Physio/ 301, 1004-1013, doi:10.1152/ajpgi.00167.2011 (2011). 
30 I-Che Chung, C.-N. O., Sheng-Ning Yuan, Hsin-Chung Lin, Kuo-Yang Huang, Pao-Shu Wu, Chia-Yuan Liu, Kuen-Jou Tsai, Lai-Keng Loi, Yu-Jen Chen, An-Ko Chung, David M. Ojcius \& , Y.-S. C., Lih-Chyang Chen. Pretreatment with a Heat-Killed Probiotic Modulates. Nutrients 11, 1-16, doi:10.3390/nu11030516 (2019).

$31 \mathrm{Hu}$, J. et al. Anti-tumour immune effect of oral administration of Lactobacillus plantarum to CT26 tumour-bearing mice. J Biosci 40, 269-279, doi:10.1007/s12038-015-9518-4 (2015).

$32 \mathrm{Ma}$, E. L. et al. The anticancer effect of probiotic Bacillus polyfermenticus on human colon cancer cells is mediated through ErbB2 and ErbB3 inhibition. Int J Cancer 127, 780-790, doi:10.1002/ijc.25011 (2010).

33 Kumar, A., Singh, N. K. \& Sinha, P. R. Inhibition of 1,2-dimethylhydrazine induced colon genotoxicity in rats by the administration of probiotic curd. Mol Biol Rep 37, 1373-1376, doi:10.1007/s11033-009-9519-1 (2010).

34 Yuanchun Yuea, K. Y., Jing Lu, Xinyu Wang, Shuwen Zhang, Liu Liu, Baoyu Yang, Khaled Nassar, Xiaoxi Xua, Xiaoyang Pang, Jiaping Lv. Probiotic strain Lactobacillus plantarum YYC-3 prevents colon cancer in mice. Biomedicine \& Pharmacotherapy 127, 1-8, doi:10.1016/j.biopha.2020.110159 (2020).

35 Hold, G. L. Gastrointestinal Microbiota and Colon Cancer. Dig Dis 34, 244-250, doi:10.1159/000443358 (2016).

36 Cullin, N., Azevedo Antunes, C., Straussman, R., Stein-Thoeringer, C. K. \& Elinav, E. Microbiome and cancer. Cancer Cel/ 39, 1317-1341, doi:10.1016/j.ccell.2021.08.006 (2021).

37 Shiao, S. L. et al. Commensal bacteria and fungi differentially regulate tumor responses to radiation therapy. Cancer Cel/ 39, 1202-1213 e1206, doi:10.1016/j.ccell.2021.07.002 (2021).

38 Reichardt, N. et al. Phylogenetic distribution of three pathways for propionate production within the human gut microbiota. ISME J 8, 1323-1335, doi:10.1038/ismej.2014.14 (2014).

39 Scheiman, J. et al. Meta-omics analysis of elite athletes identifies a performance-enhancing microbe that functions via lactate metabolism. Nat Med 25, 1104-1109, doi:10.1038/s41591-019-0485-4 (2019).

40 Ley, R. E. Gut microbiota in 2015: Prevotella in the gut: choose carefully. Nat Rev Gastroenterol Hepatol 13, 69-70, doi:10.1038/nrgastro.2016.4 (2016).

41 Lukens, J. R. et al. Dietary modulation of the microbiome affects autoinflammatory disease. Nature 516, 246-249, doi:10.1038/nature13788 (2014).

42 Ou, J., DeLany, J. P., Zhang, M., Sharma, S. \& O'Keefe, S. J. Association between low colonic shortchain fatty acids and high bile acids in high colon cancer risk populations. Nutr Cancer 64, 34-40, doi:10.1080/01635581.2012.630164 (2012). 
43 Bernstein, H., Bernstein, C., Payne, C. M. \& Dvorak, K. Bile acids as endogenous etiologic agents in gastrointestinal cancer. World J Gastroentero/ 15, 3329-3340, doi:10.3748/wjg.15.3329 (2009).

44 Pyne, N. J. \& Pyne, S. Sphingosine 1-phosphate and cancer. Nat Rev Cancer 10, 489-503, doi:10.1038/nrc2875 (2010).

45 Babak Oskouian, P. S., Alexander D. Borowsky, Angelina Crans, Lisa Dillard-Telm, \& Yuen Yee Tam, P. B., and Julie D. Saba. Sphingosine-1-phosphate lyase potentiates apoptosis via p53- and p38-dependent pathways and is down-regulated in colon cancer. PNAS 103, 17384-17389, doi:10.1073pnas.0600050103 (2006).

46 Visentin, B. et al. Validation of an anti-sphingosine-1-phosphate antibody as a potential therapeutic in reducing growth, invasion, and angiogenesis in multiple tumor lineages. Cancer Cell 9, 225-238, doi:10.1016/j.ccr.2006.02.023 (2006).

47 Besim Ogretmen, Y. A. H. BIOLOGICALLY ACTIVE SPHINGOLIPIDS IN CANCER PATHOGENESIS AND TREATMENT. Nat Rev Cancer 4, 604-616, doi:10.1038/nrc1411 (2004).

48 Huwiler, A. \& Pfeilschifter, J. New players on the center stage: sphingosine 1-phosphate and its receptors as drug targets. Biochem Pharmacol 75, 1893-1900, doi:10.1016/j.bcp.2007.12.018 (2008).

49 Slizewska, K., Markowiak-Kopec, P. \& Slizewska, W. The Role of Probiotics in Cancer Prevention. Cancers (Basel) 13, doi:10.3390/cancers13010020 (2020).

50 Julia B. Ewaschuk, J. W. W., Hugo Diaz, Karen L. Madsen. Bioproduction of Conjugated Linoleic Acid by Probiotic Bacteria Occurs In Vitro and In Vivo in Mice.pdf. The Journal of nutrition 136, 1483-1487, doi:10.1093/jn/136.6.1483 (2006).

51 Siddiqui, A. \& Ceppi, P. A non-proliferative role of pyrimidine metabolism in cancer. Mol Metab 35, 100962, doi:10.1016/j.molmet.2020.02.005 (2020).

52 Zhang, W. C. et al. Glycine decarboxylase activity drives non-small cell lung cancer tumor-initiating cells and tumorigenesis. Cell 148, 259-272, doi:10.1016/j.cell.2011.11.050 (2012).

\section{Figures}




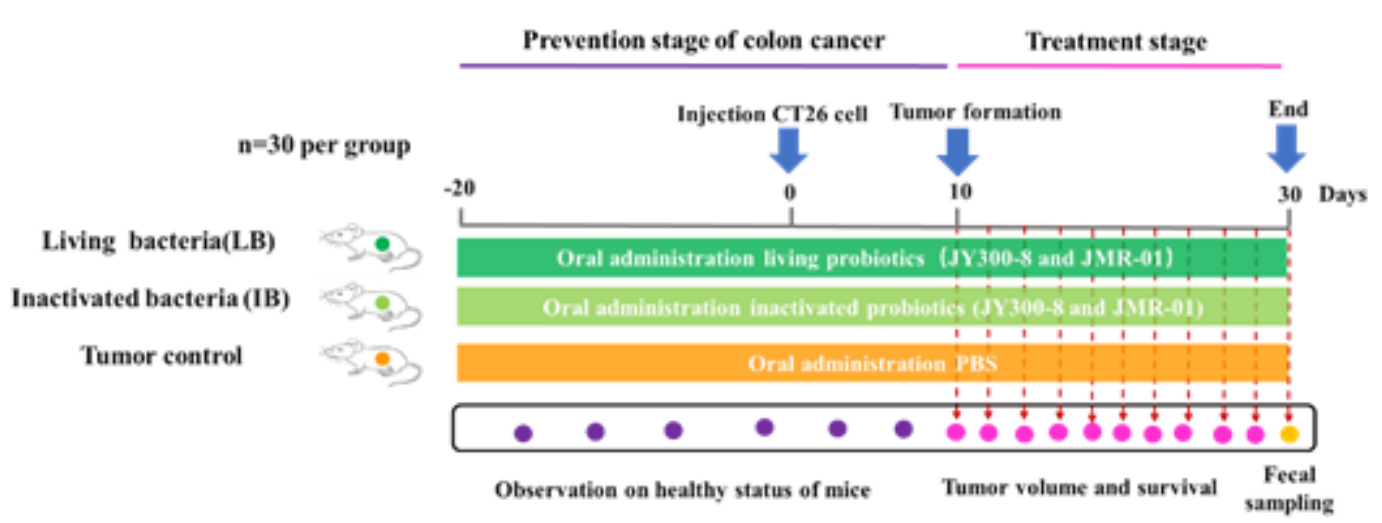

b

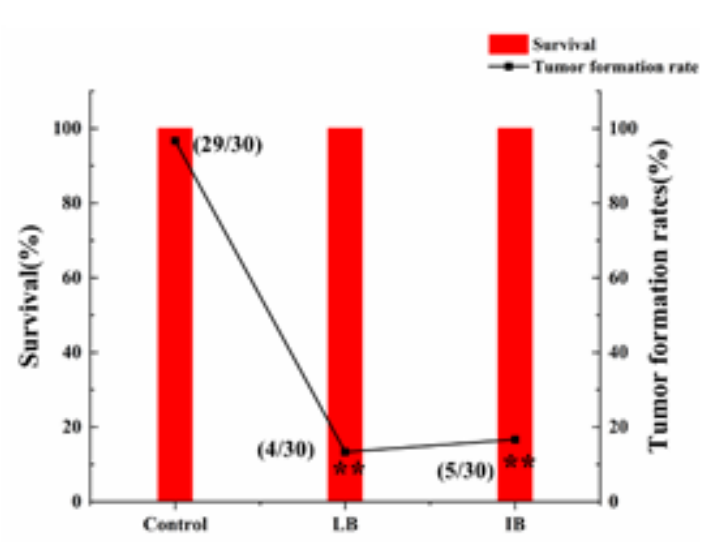

d

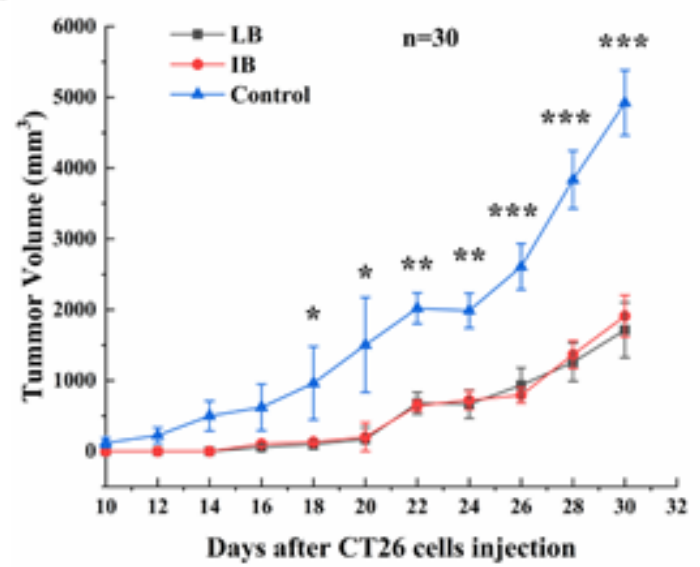

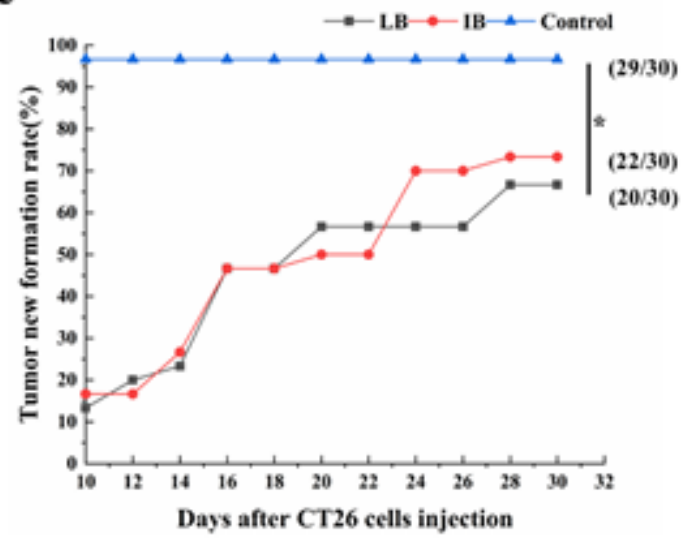

e

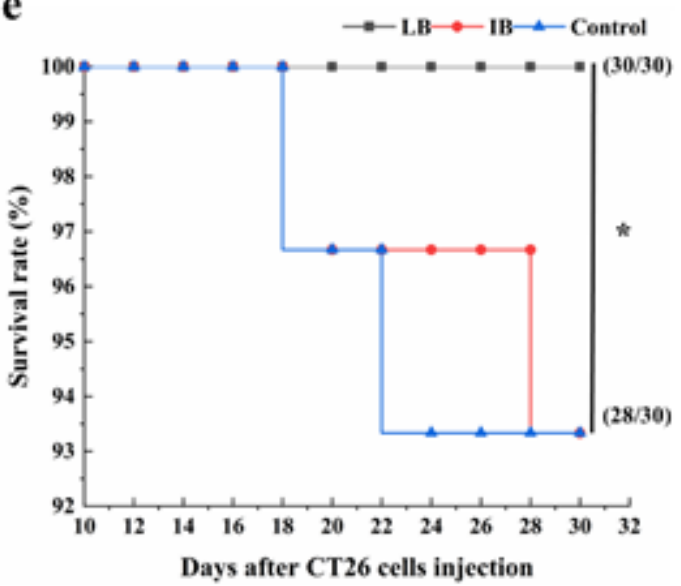

Figure 1

Commensal Lactobacillus suppressed the occurrence and development of colon cancer induced by injection of CT-26 cells in mice. (a The experimental scheme for tumor prevention and treatment by oral administration of JY300-8 and JMR-01. Mice ( $n=30$ per group) were administrated with LB, IB, and PBS for 20 days in advance, then injected the CT26 cells subcutaneously, followed by schemed treatment for 10 days until the $100 \mathrm{~mm}^{3}$ of mean volume were reached in tumor control, then the survival and tumor formation rates were assessed. Subsequently, schedules for the measurement of tumor volume, survival, tumor new formation rate and fecal sampling are indicated with arrows. $\mathbf{b}$ The survival and tumor formation rates in three groups at 10 days when mice in control were successfully established colon cancer model, ${ }^{\star *} \mathrm{P}<0.01$ for control vs LB, control vs IB. $\mathbf{c}$ The tumor new formation rates were assessed 
during the experiment. $\mathbf{d}$ The whole tumor growth kinetics in bearing-tumor BABL/C mice after colon cancel model establishment (means \pm SEM), ${ }^{P} P<0.05,{ }^{*} \mathrm{P}<0.01$, ${ }^{\star \star *} \mathrm{P}<0.001$ for control vs. LB/IB. e The survival of bearing tumor mice in all groups.)

a

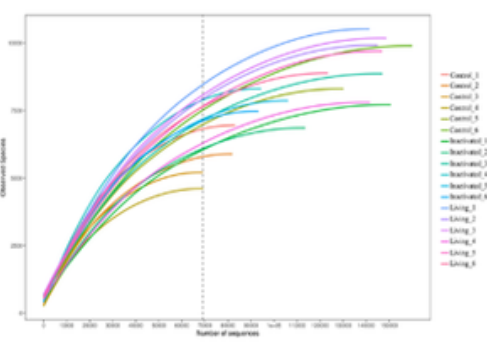

c

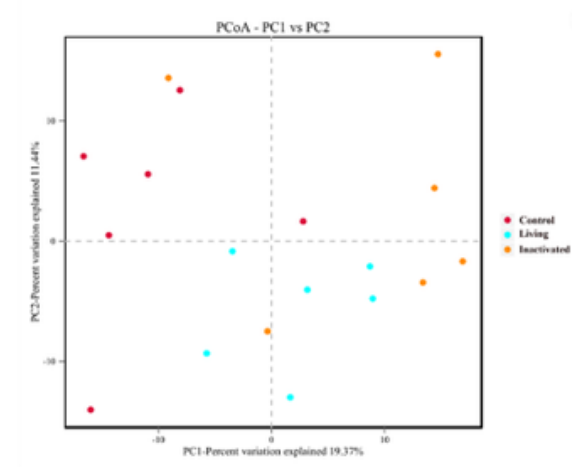

e

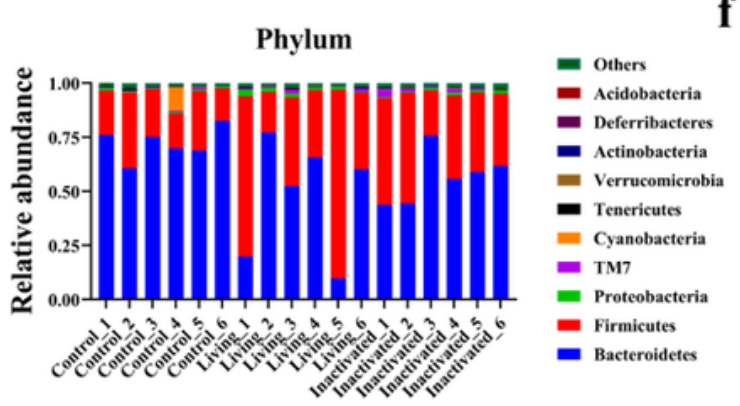

g

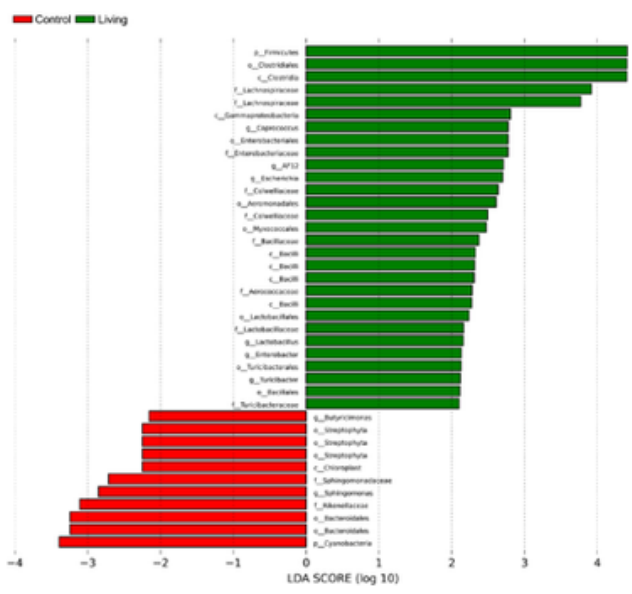

b

d

f
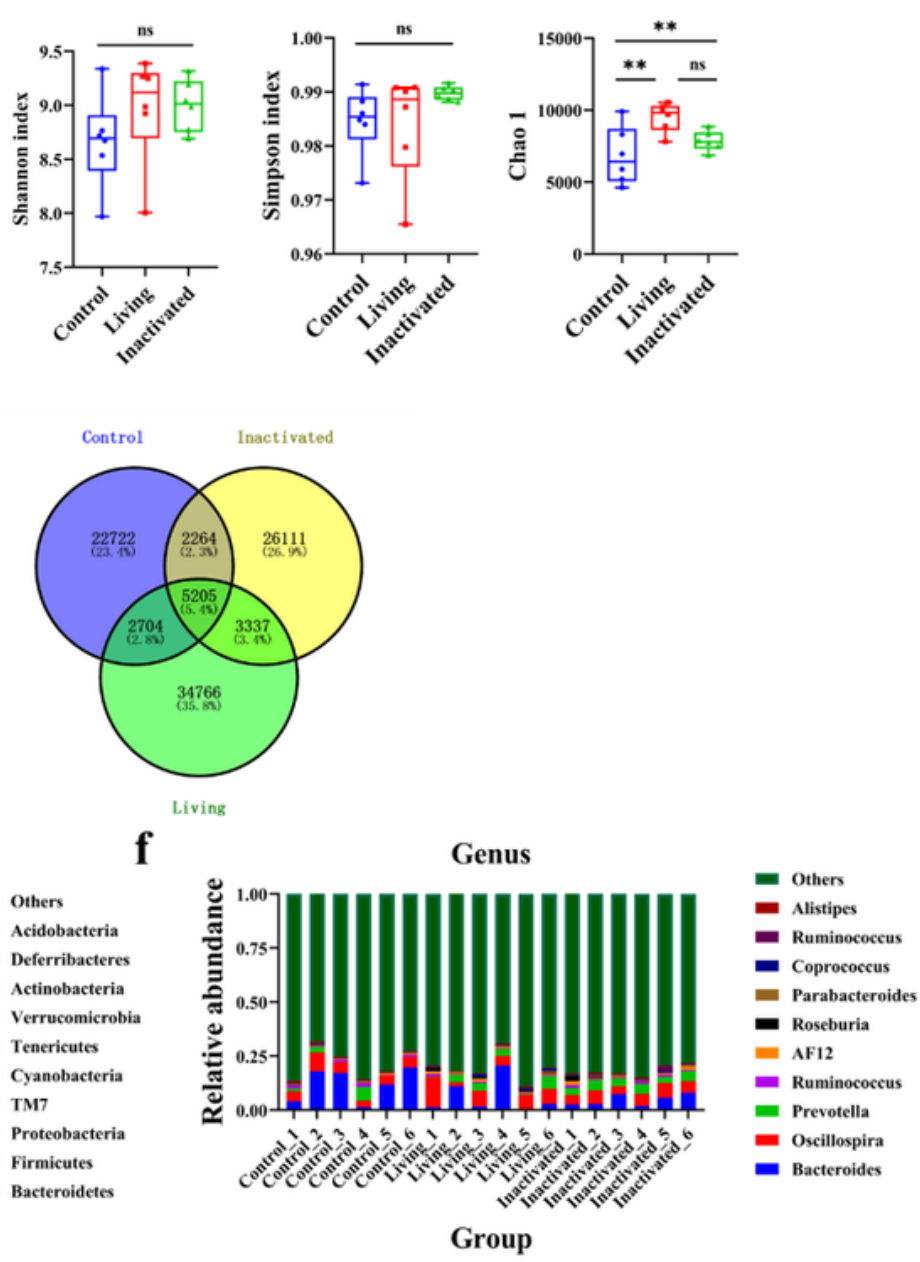

- control
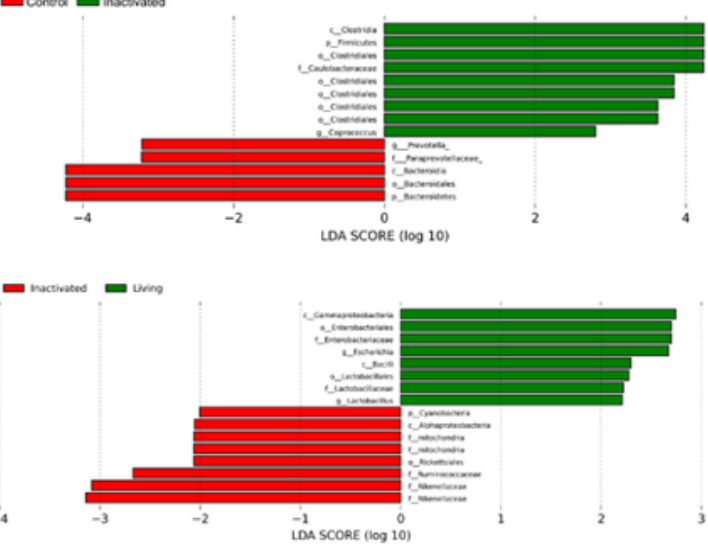

Figure 2 


\section{Longitudinal analyses of the gut microbiota of tumor mice treated with commensal Lactobacillus (a The}

rarefaction curve of microbial communities in the fecal samples. $\mathbf{b}$ Changes in alp.ha diversity indices of microbial communities in the fecal samples, including Shannon, Simpson and Chao 1 indices. c Principal coordinate analysis based on Bray-Curtis dissimilarity. Each dot indicates a single sample and each group was shown in a different color. $\mathbf{d}$ Venn diagram analysis of OTU overlaps between different groups of microorganisms. e Relative abundance at the phylum level. $\mathbf{f}$ Relative abundance at the genus level. $\mathbf{g}$ Linear discriminant analysis (LDA) effect size of fecal samples after various treatment. The red or green columns in the histogram represented the microbial groups that played a vital role in their group, respectively. The figure showed only microbiota whose LDA score was greater than 2 . The length of the bar chart represented the size of the LDA value.) 
a

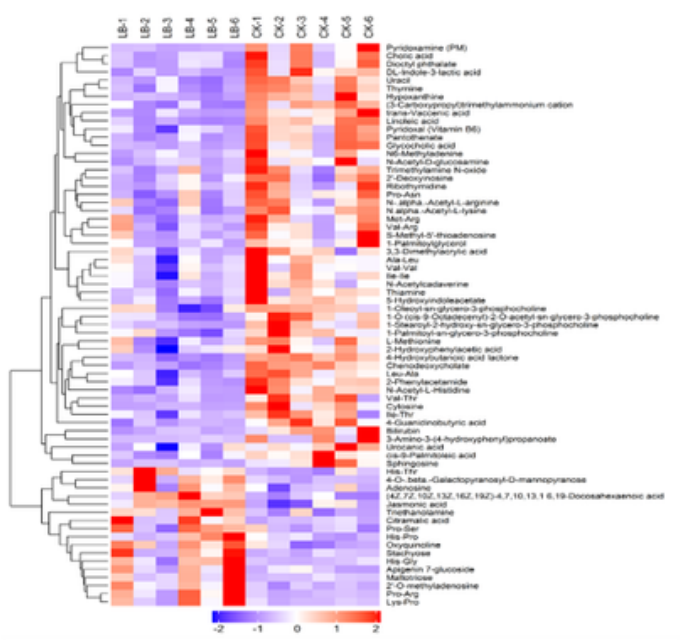

b

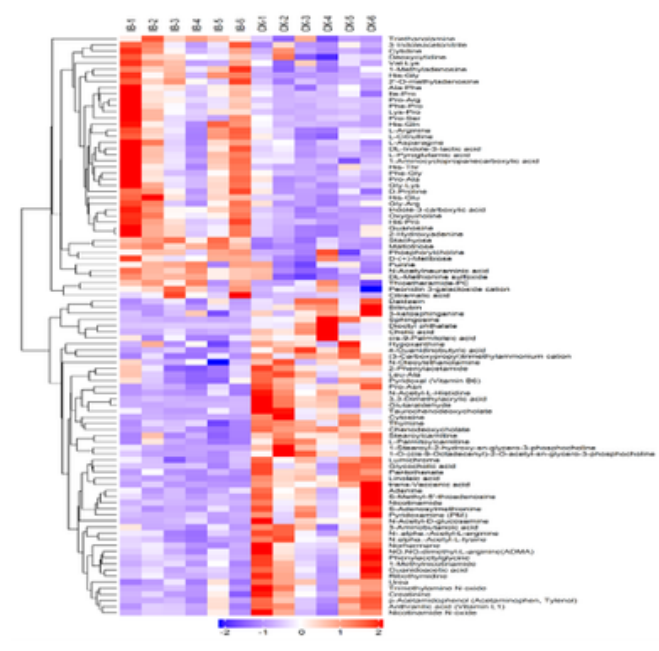

c

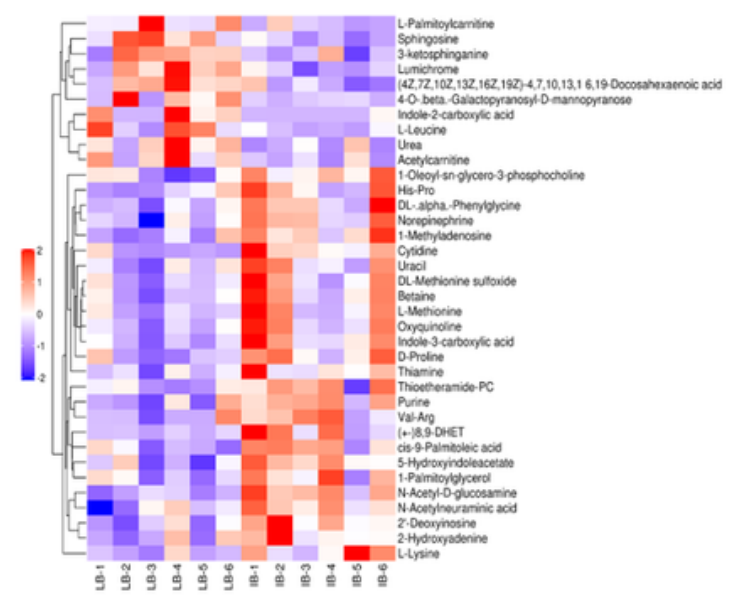

Negative
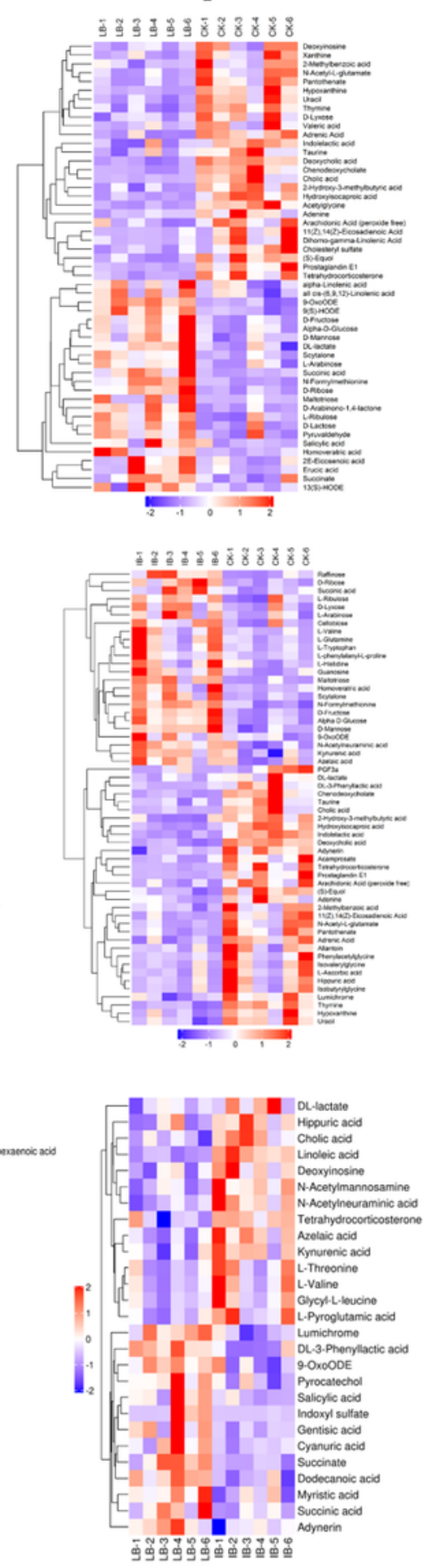

Figure 3

Metabolic profiles analysis of fecal samples in mice with different treatments (Cluster analysis of differential metabolites for LB vs tumor control (CK) (a), IB vs control (CK) (b) and LB vs IB (c) in positive and negative ions mode, respectively. Each row represented a differential metabolite, each column showed a sample, and the color represented the amount of expression, blue to red corresponding to the amount of expression from low to high.) 
a
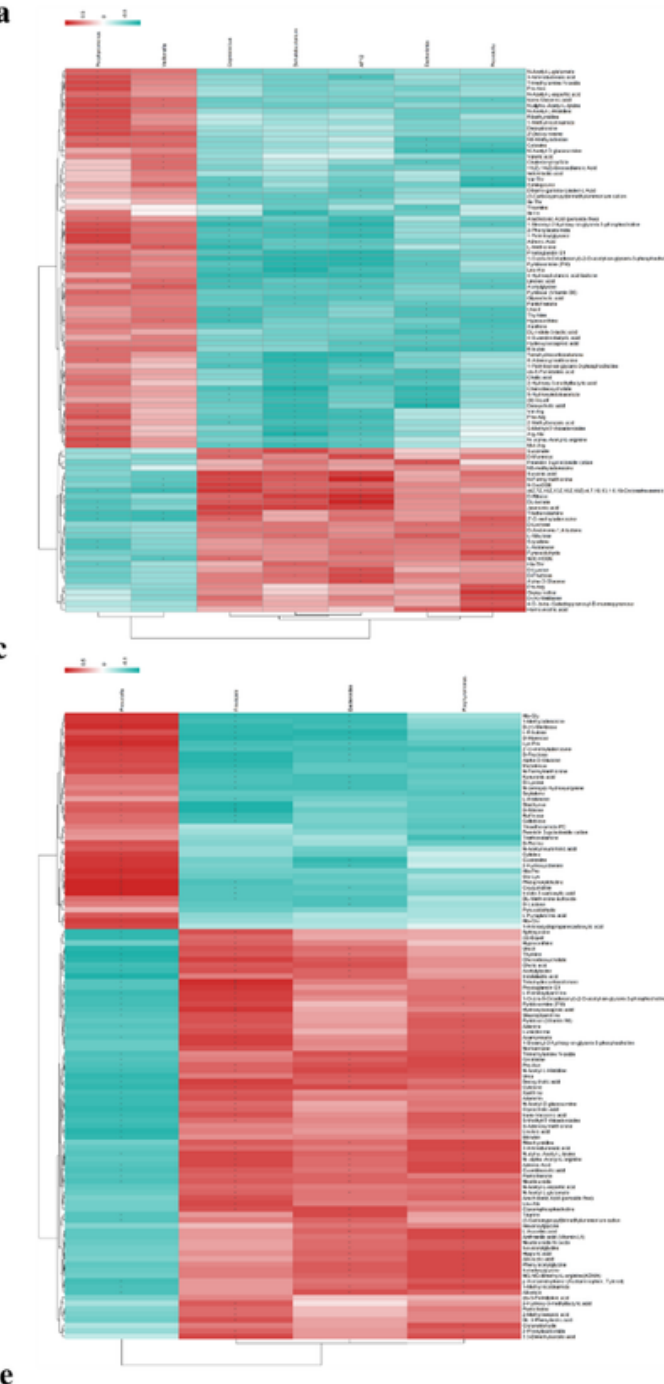

e

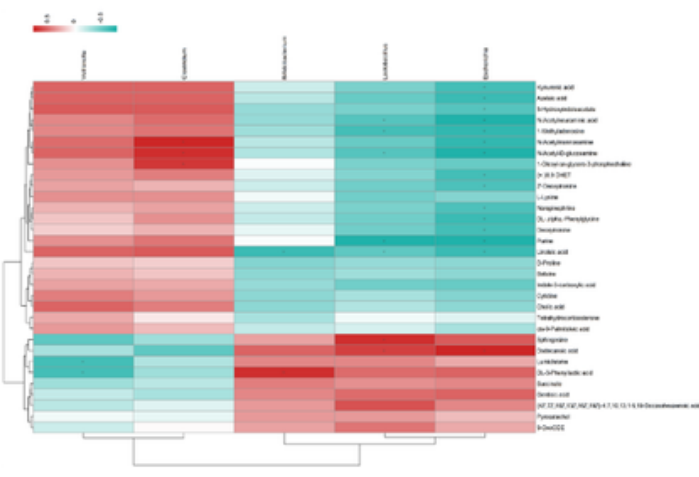

b

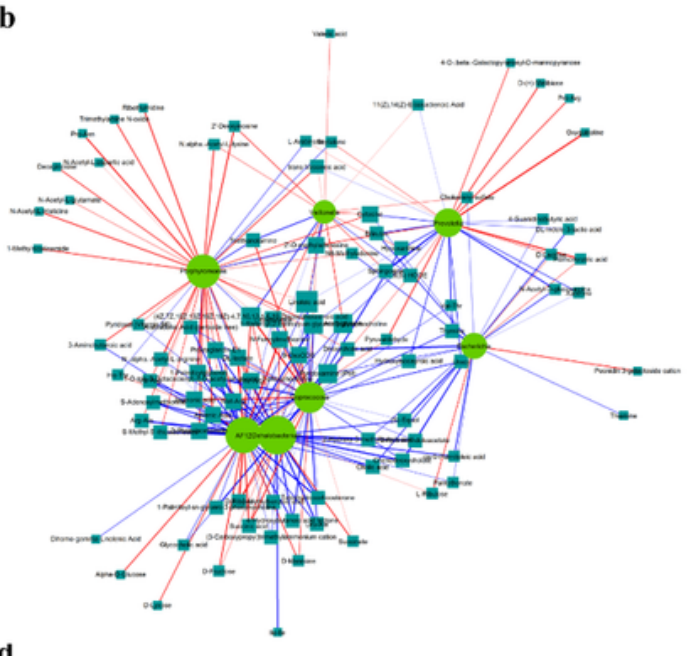

d
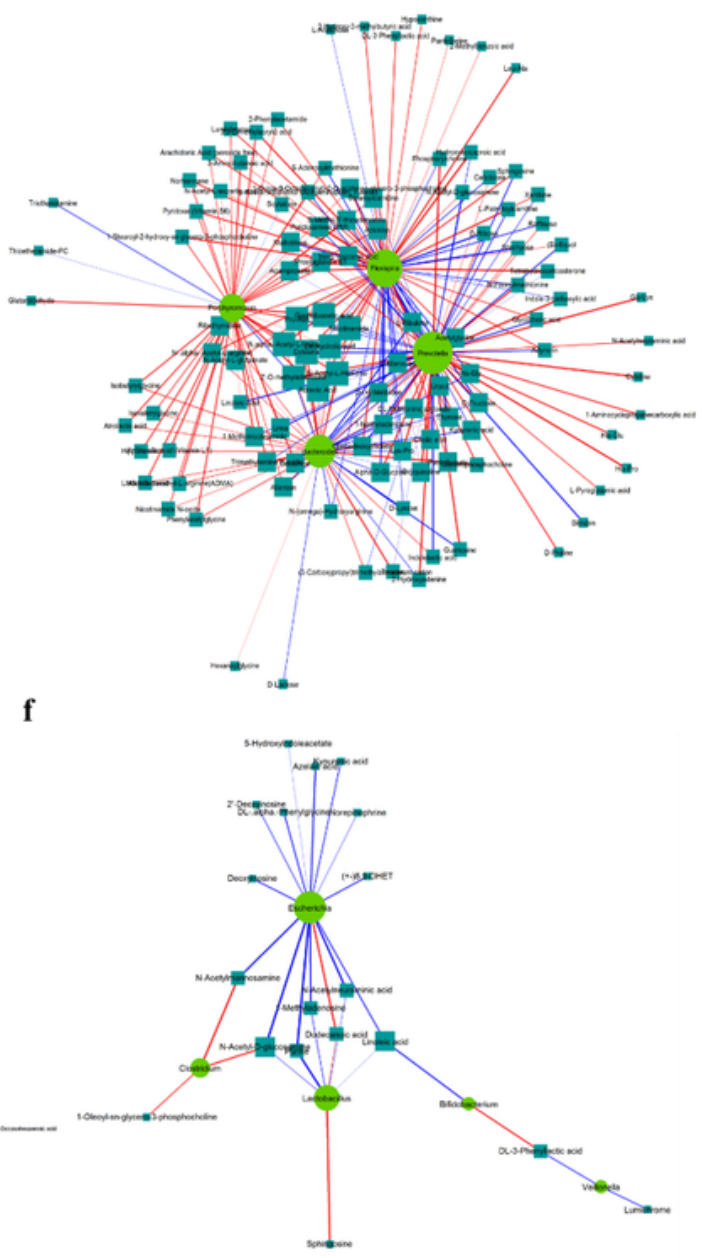

\section{Figure 4}

Correlation analysis of $16 \mathrm{~S}$ microbial diversity and metabolomics with different treatments (The hierarchical cluster heat map of spearman correlation analysis on significant difference microbiota and metabolites in control_LB groups (a), control_IB groups (c) and LB_IB groups(e), respectively. Each row in the hierarchical cluster heat map represented a significant difference metabolite and each column represented a significant difference microbiota at genus level. The correlation coefficient $\mathrm{R}$ was shown in 
color. $\mathrm{R}>0$ was positive correlation, shown in red; $\mathrm{R}<0$ represented a negative correlation, shown in blue, and the deeper the color were corresponding to the stronger the correlation. The network diagram of spearman correlation analysis on microbiota and metabolites with significant differences in control_LB groups (b), control_IB groups (d), and LB_IB groups (f), respectively, in which the circle represented bacteria with significant differences and the rectangle represented metabolites with significant differences. The color of the line represented the value of the correlation each other (blue represented negative correlation, red represented positive correlation), and the thickness of the line was proportional to the absolute value of the correlation coefficient. Node size was positively correlated with its degree, namely, the larger the degree was associated with the larger the node size.)

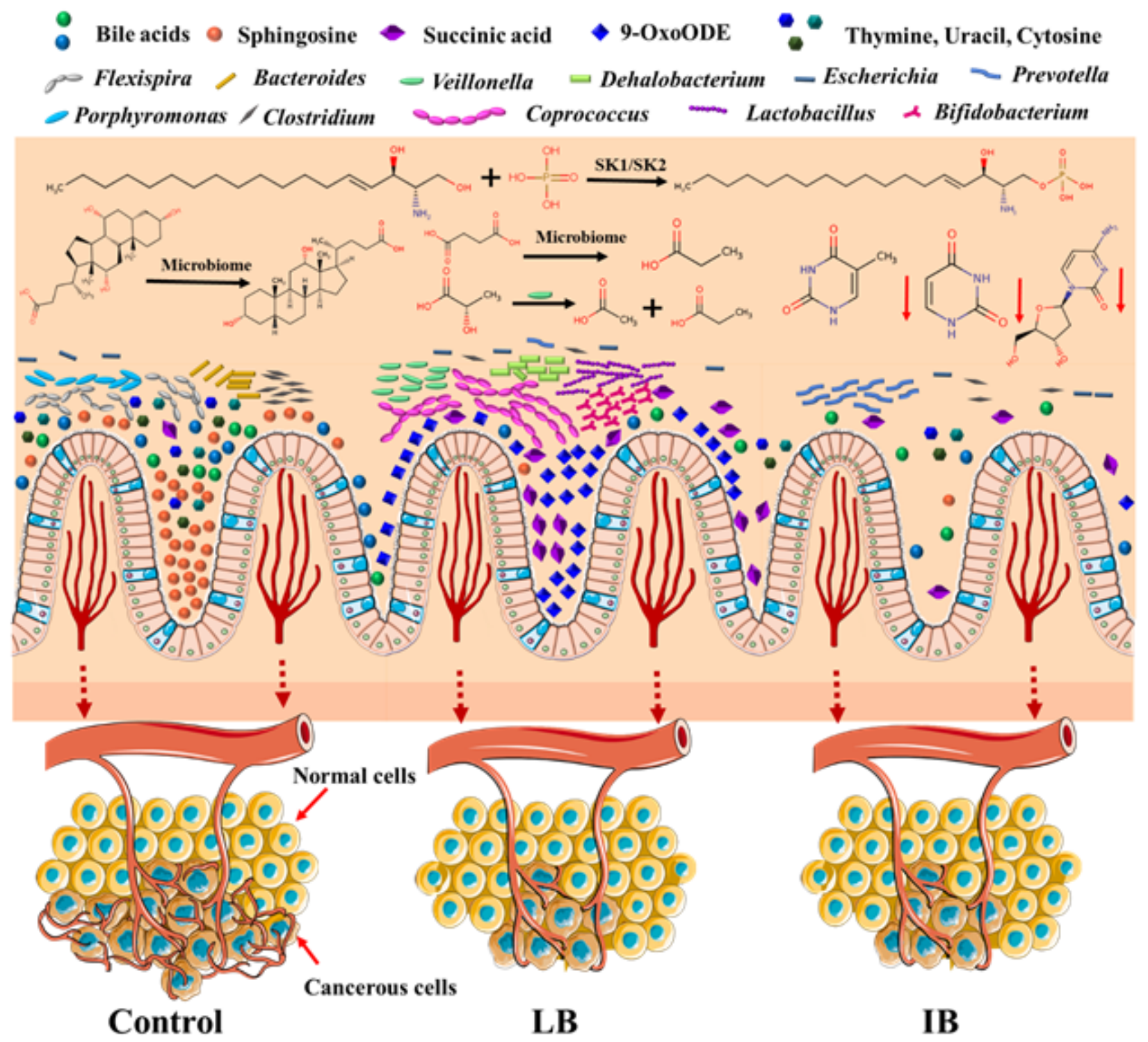

Figure 5

Graphical representation of major microbial and metabolomic alterations correlated with colon cancer progression using various treatment. 


\section{Supplementary Files}

This is a list of supplementary files associated with this preprint. Click to download.

- AdditionalFiles.rar 